\title{
Encapsulation of a Chloroform Molecule in a Peptide Nanotube
}

\author{
Fainida Rahmat ${ }^{1}$, Ngamta Thamwattana ${ }^{2}$ \\ ${ }^{1}$ Department of Mathematics, Faculty of Science and Mathematics, Sultan Idris University of Education, Perak, \\ Malaysia \\ ${ }^{2}$ School of Mathematics and Applied Statistics, University of Wollongong, Wollongong, Australia \\ Email: fainida@fsmt.upsi.edu.my, ngamta@uow.edu.au
}

Received 8 June 2014; revised 19 July 2014; accepted 5 August 2014

Copyright (C) 2014 by authors and Scientific Research Publishing Inc.

This work is licensed under the Creative Commons Attribution International License (CC BY).

http://creativecommons.org/licenses/by/4.0/

(c) (i) Open Access

\begin{abstract}
We determine the encapsulation of a chloroform molecule into a D,L-Ala cyclopeptide nanotube by investigating the interaction energy between the two molecular structures. We employ the Lennard-Jones potential and a continuum approach which assumes that the atoms are evenly distributed over the molecules providing average atomic densities. Our result demonstrates that the encapsulation depends on the size of the molecule and the internal diameter of the peptide nantube. In particular, the on-axis chloroform molecule is only accepted into a peptide nanotube whose internal radius is greater than $5 \AA$. If located near the edge of the nanotube, then it is unlikely that the chloroform molecule will enter the nanotube. This is due to the energy valley that the molecule will need to overcome to move past the edge into the open end of the nanotube.
\end{abstract}

\section{Keywords}

Peptide Nanotubes, Chloroform, Lennard-Jones Potential, Van Der Waals Interaction

\section{Introduction}

Peptide nanotubes have promising applications in many fields, such as chemical, biological, medical and material sciences [1]. Peptide nanotubes consist of cyclic peptide rings with an even number of alternating L-D amino acid residues. These cyclic peptide rings adopt flat-ring shaped conformation with C-O and N-H bonds that are perpendicular to the plane of the ring with the side chain of the amino acids pointing outward. In a suitable condition, these rings self-assemble to form hollow tubular structures as the rings stack on top of each other through the network of hydrogen bonding between adjacent rings [2]-[5].

One of the proposed applications for peptide nanotubes is as artificial transmembrane channels that have the 
capability to mimic naturally occurring ion channels for transporting ions, water and small molecules across cell membranes [6] [7]. Since the experimental observation of Ghadiri et al. [6] on the transport activities of $\mathrm{K}^{+}$and $\mathrm{Na}^{+}$in peptide nanotubes, many studies investigate various aspects of peptide nanotubes as potential transmembrane channels [5] [7]-[15]. Several studies investigate the permeation of ions into peptide nanotubes and determine the behaviour of the ions inside the tubes based on the energy profiles [9] [10] [12]. There are also studies investigating the dynamics and the distribution of water molecules in peptide nanotubes using molecular dynamics simulations [5] [13] [14]. In Cheng et al. [11], the adsorption of a chloroform molecule $\left(\mathrm{CHCl}_{3}\right)$ onto the surface of a cyclo[(1 R, $3 \mathrm{~S})-\gamma$-Acc-D-Phe $]_{3}$ peptide nanotube in a solvent box of $100 \mathrm{CHCl}_{3}$ is studied by employing molecular dynamics simulations. They find that $\mathrm{CHCl}_{3}$ molecules can bind to both the outer surface and the inner lumen of the peptide nanotube. Based on these results, Cheng et al. [11] suggest that peptide nanotubes are capable of transporting $\mathrm{CHCl}_{3}$ molecules, demonstrating their potential use as artificial channels for organic molecules.

To further our understanding of the interaction between chloroform molecules and peptide nanotubes, this paper develops mathematical models to describe the interaction between the two structures, aiming to elucidate the encapsulation behaviour of the chloroform molecules into peptide nanotubes. Two scenarios are considered, namely: 1) an on-axis chloroform molecule and 2) a chloroform molecule located near the edge of the peptide nanotube. We employ the Lennard-Jones potential and the continuum approach to derive an analytical expression for the interaction energy between a chloroform molecule and a peptide nanotube. This model can be used to determine the condition under which the chloroform molecule will be encapsulated into a peptide nanotube. By minimizing the interaction energy we can also predict its preferred location inside the nanotube. We note that this model can be adopted to study other spherical molecular structures interacting with peptide nanotubes.

This paper is structured as follows. In Section 2, we briefly introduce the Lennard-Jones potential and the continuum approach. In Sections 3 and 4, we derive analytical expressions for the potential energy for the two assumed locations of the chloroform molecule interacting with a peptide nanotube. In the subsequent section we present the numerical results and discussion. Finally, we summarize our findings in Section 6.

\section{Modelling Approach}

To obtain the interaction energy between a chloroform molecule and a peptide nanotube, we use the LennardJones potential and a continuum approach. The continuum approach using the Lennard-Jones potential was first introduced by Girifalco [16] for the interaction between two $\mathrm{C}_{60}$ molecules. Subsequently, this approach has been successfully adopted by many researchers, such as Baowan et al. [17] [18] and Cox et al. [19] [20] to obtain explicit analytical criteria for the interaction potentials involving carbon nanostructures. Furthermore, studies of interactions involving biological materials such as lipid bilayers, lipid nanotubes and liposomes [21]-[23], have also indicated that this approach produces results that are in good agreement with intensive computational studies. For the study of peptide nanotubes, Rahmat et al. [24] adopt the Lennard-Jones potential and the continuum approach to model the interactions of a peptide nanotube with an ion, an ion-water cluster and a fullerene $\mathrm{C}_{60}$. The results obtained are shown to be consistent with experiments and molecular dynamics simulations. With this in mind, this paper extends the modelling approach in [24] to investigate the potential use of peptide nanotubes as an artificial channel for transporting organic molecules, such as chloroform.

The Lennard-Jones potential for two nonbonded atoms at a distance $\rho$ apart is given by

$$
\Phi(\rho)=-A \rho^{-6}+B \rho^{-12},
$$

where $A$ and $B$ represent the attractive and repulsive constants, respectively. We can also write this equation as

$$
\Phi(\rho)=4 \varepsilon\left[\left(\frac{\sigma}{\rho}\right)^{12}-\left(\frac{\sigma}{\rho}\right)^{6}\right],
$$

where $\varepsilon$ is the energy well depth and $\sigma$ is the van der Waals diameter. The repulsive term $1 / \rho^{12}$ dominates at a short distance, while the attractive term $1 / \rho^{6}$ dominates at a longer distance.

The conventional pairwise method determines the interaction energy for two nanostructures by summing the potential energy for each atom pair, namely

$$
E=\sum_{i} \sum_{j} \Phi\left(\rho_{i j}\right)=\sum_{i} \sum_{j}\left(-A_{i j} \rho_{i j}^{-6}+B_{i j} \rho_{i j}^{-12}\right),
$$


where the constants $A_{i j}$ and $B_{i j}$ for the interaction between atoms $i$ and $j$ are calculated from $A_{i j}=4 \varepsilon_{i j} \sigma_{i j}^{6}$ and $B_{i j}=4 \varepsilon_{i j} \sigma_{i j}^{12}$ where $\sigma_{i j}=\left(\sigma_{i}+\sigma_{j}\right) / 2$ and $\varepsilon_{i j}=\left(\varepsilon_{i} \varepsilon_{j}\right)^{1 / 2}$.

In a continuum approach, atoms are assumed to be evenly distributed over the entire surface of the nanostructure. Thus, we may write the potential energy as a double integral over the surfaces of each nanostructure,

$$
E=\mu_{1} \mu_{2} \int_{S_{1}} \int_{S_{2}} \Phi(\rho) \mathrm{d} S_{1} \mathrm{~d} S_{2},
$$

where $\mu_{1}$ and $\mu_{2}$ denote the mean surface densities of atoms on each nanostructure, and $\rho$ is the distance between typical surface elements $\mathrm{d} S_{1}$ and $\mathrm{d} S_{2}$ on the two different nanostructures.

To adopt the continuum approach, we consider the chloroform molecule to be a sphere, where the $\mathrm{C}$ atom is assumed to be at the centre of the sphere and the $\mathrm{Cl}$ and $\mathrm{H}$ atoms are assumed to be on the surface of the sphere. Further, we assume that a peptide nanotube is made up of $n$ units, where each unit comprises an inner and an outer tube. For the study of the chloroform molecules, Cheng et al. [11] use the cyclo[(1 R, $3 \mathrm{~S})-\gamma$-Acc-D-Phe $]_{3}$ peptide nanotube. However, in this study we adopt the cyclo[(-D-Ala-L-Ala $\left.)_{4}-\right]$ peptide nanotube since it has a simple molecular structure. From [1], each cyclo[(-D-Ala-L-Ala) $\left.4^{-}\right]$peptide unit has eight amino acid residues. The inner tube consists of 48 backbone atoms which are made up of 16 carbon atoms, 16 hydrogen atoms, 8 nitrogen atoms and 8 oxygen atoms whilst the outer tube consists of 32 atoms including 8 carbon atoms and 24 hydrogen atoms. The radii of the inner and the outer tube for cyclo[(-D-Ala-L-Ala) $4_{4}$ - $]$ are $4.25 \AA$, and $7.95 \AA$, respectively, $L=2.5 \AA$ is the length of each peptide unit and $\mathrm{d}=4.8 \AA$, is the average distance between the centres of two neighbouring units [1]. We also examine D, L-Ala cyclopeptide nanotubes that have 10 and 12 amino acids where based on [25] and [26], we assume that the inner tube radii are $5 \AA$ and $6.5 \AA$, respectively. The numerical values for constants used in this paper are given in Table 1 , where the van der Waals parameters, $\sigma_{i}$ and $\varepsilon_{i}$, are taken from [27].

We comment that peptide nanotubes are complex structures, therefore using our approach we sacrifice some accuracy to achieve computational efficiency. However, we note in many areas the sacrifice is justified as the model captures the dominant interactions.

\section{Interaction between an on-Axis Chloroform Molecule and a Peptide Nanotube}

In this section, we investigate the interaction between an on-axis chloroform molecule and a peptide nanotube, as shown in Figure 1. We denote by $a_{1}$ and $a_{2}$ the radii of the inner and outer tubes respectively, by $L$ the length of each peptide unit and by $d$ the distance between the centres of two neighbouring units. We also assume that the spherical chloroform has radius $b$ and its centre is located at the distance $Z$ along the central axis of the peptide nanotube.

\begin{tabular}{ccc} 
Table 1. Numerical values of constants used in the model. & \\
\hline Interaction & $A\left(\AA^{6} \times \mathrm{kcal} / \mathrm{mol}\right)$ & $B\left(\AA^{12} \times \mathrm{kcal} / \mathrm{mol}\right)$ \\
\hline C-C & 684.95 & $1.12 \times 10^{6}$ \\
C-H & 198.60 & $1.45 \times 10^{5}$ \\
C-N & 477.59 & $6.70 \times 10^{5}$ \\
C-O & 391.38 & $4.82 \times 10^{5}$ \\
C-Cl & 1084.82 & $1.91 \times 10^{6}$ \\
H-H & 50.85 & $1.47 \times 10^{6}$ \\
H-N & 135.47 & $8.32 \times 10^{6}$ \\
H-O & 108.90 & $5.77 \times 10^{6}$ \\
H-Cl & 317.88 & $2.53 \times 10^{5}$ \\
N-Cl & 757.82 & $1.15 \times 10^{6}$ \\
O-Cl & 622.05 & $8.29 \times 10^{5}$ \\
\hline
\end{tabular}




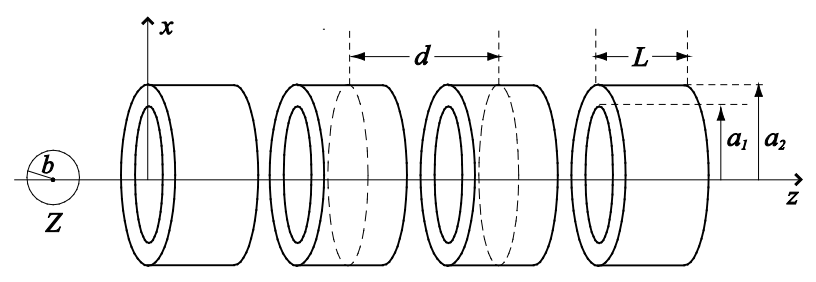

Figure 1. Interaction between an on-axis chloroform molecule and a peptide nanotube.

The total interaction energy between the on-axis chloroform and the peptide nanotube comprises four components: 1) carbon atom at the centre of the sphere and the inner tube; 2) carbon atom at the centre of the sphere and the outer tube; 3) $\mathrm{Cl}$ and $\mathrm{H}$ atoms on the surface of the sphere and the inner tube tube, and 4) $\mathrm{Cl}$ and $\mathrm{H}$ atoms on the surface of the sphere and the outer tube. Thus, we have

$$
E=E_{A I}+E_{A O}+E_{S I}+E_{S O},
$$

where $E_{A I}, E_{A O}, E_{S I}$ and $E_{S O}$ are the interaction energies corresponding to (1)-(4), respectively. Following [20], the interaction between an atom and a nanotube can be written as

$$
E_{A T}=-2 \pi a \mu_{t}\left(-A P_{6}+B P_{12}\right),
$$

where $P_{n}$ is given by

$$
P_{n}=-\frac{1}{a^{2 n+1}} \int_{\psi_{1}}^{\psi_{2}} \cos ^{2 n} \psi \mathrm{d} \psi,
$$

where $\psi_{1}=\arctan (Z / a), \psi_{2}=\arctan ([Z-L] / a), a$ is the radius of the tube and $L$ is the length of the nanotube unit and $A$ and $B$ are the average attractive and repulsive constants respectively for the interaction involving an atom and all atoms comprising the nanotube. We note that we may use

$$
\int \cos ^{2 n} \psi \mathrm{d} \psi=\frac{1}{2^{2 n}}\left[\left(\begin{array}{c}
2 n \\
n
\end{array}\right) \psi+\sum_{i=0}^{n-1}\left(\begin{array}{c}
2 n \\
i
\end{array}\right) \frac{\sin (2(n-i) \psi)}{n-i}\right],
$$

to obtain an analytical solution of (3.2). The mean atomic surface density $\mu_{t}$ for either the inner or the outer tube is calculated as $\mu_{\mathrm{t}}=$ (number of atoms) $/ 2 \pi a L$.

From [20], the interaction between a sphere and a nanotube is given by

$$
E_{S T}=8 \pi^{2} a b^{2} \mu_{s} \mu_{t}\left\{-A\left[Q_{2}+2 b^{2} Q_{3}\right]+\frac{B}{5}\left[5 Q_{5}+80 b^{2} Q_{6}+336 b^{4} Q_{7}+512 b^{6} Q_{8}+256 b^{8} Q_{9}\right]\right\},
$$

where

$$
Q_{n}=-\frac{1}{\lambda^{2 n+1}} \int_{\psi_{1}}^{\psi_{2}} \cos ^{2 n} \psi \mathrm{d} \psi,
$$

and $\mu_{s}=$ (number of atoms on the sphere) $/\left(4 \pi b^{2}\right), \lambda=\left(a^{2}-b^{2}\right)^{1 / 2}, \psi_{1}=\arctan (Z / \lambda)$ and $\psi_{2}=\arctan ([Z-L] / \lambda)$ and $A$ and $B$ are the average attractive and repulsive constants, respectively, for the interaction involving atoms on the surface of the sphere and atoms comprising the nanotube. We note that we can use (3.3) to evaluate the integral $Q_{n}$ in (3.5).

\section{Interaction for a Chloroform Molecule near the Edge of a Peptide Nanotube}

Here, we investigate the interaction for a chloroform molecule located near the edge of a peptide nanotube, as shown in Figure 2. The centre of the chloroform is assumed to have coordinates $(x, 0, Z)$ and a typical point on the surface of the nanotube is assumed to have coordinates $(a \cos \theta, a \sin \theta, z)$. The sphere is assumed to be rotated at an angle $\varphi$, such that $Z=r \cos \varphi$ and $x=a+r \sin \varphi$, where $r$ is the distance between the edge of the nanotube and the centre of the chloroform molecule. The distance $\rho$ between these two typical elements is given by

$$
\rho^{2}=(a \cos \theta-x)^{2}+a^{2} \sin ^{2} \theta+(z-Z)^{2}=\alpha^{2}+(z-Z)^{2},
$$




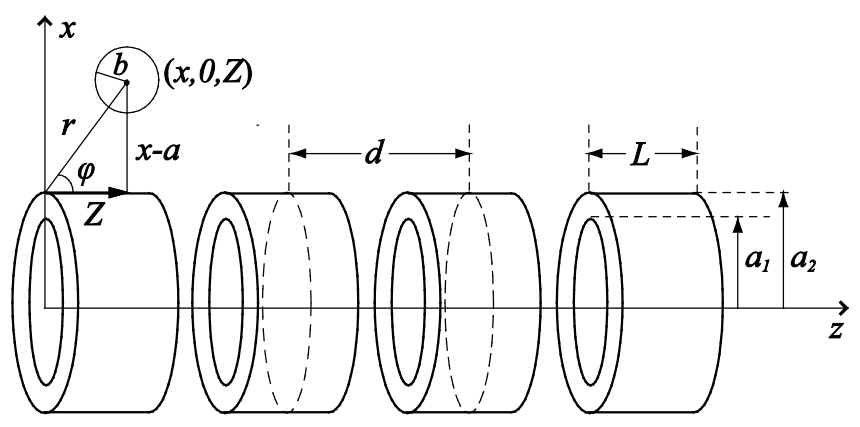

Figure 2. Interaction for a chloroform molecule near the edge of a peptide nanotube.

where $\alpha^{2}=(a+x)^{2}-4 a x \cos ^{2}(\theta / 2)$.

Similar to the previous section, the total interaction energy between the chloroform molecule and the peptide nanotube can be written as the sum of the four interactions, namely

$$
E^{*}=E_{A I}^{*}+E_{A O}^{*}+E_{S I}^{*}+E_{S O}^{*},
$$

where $E_{A I}^{*}$ and $E_{A O}^{*}$ are the interaction energies between the $C$ atom at the centre of the sphere and the inner and the outer tubes, respectively and $E_{S I}^{*}$ and $E_{S O}^{*}$ are the interaction energies between the surface of the sphere and the inner and outer tubes, respectively. The superscript * is used in this case to denote the energy for the chloroform molecule being at the edge of a peptide nanotube.

The interaction energy between an atom around the edge of the nanotube can be written as

$$
E_{A T}^{*}=a \mu_{t}\left(-A R_{6}+B R_{12}\right),
$$

where the integral $R_{2 n}$ is defined by

$$
R_{2 n}=\int_{-\pi}^{\pi} \int_{0}^{L} \frac{1}{\left[\alpha^{2}+(z-Z)^{2}\right]^{n}} \mathrm{~d} z \mathrm{~d} \theta,
$$

and can be evaluated to yield

$$
R_{2 n}=\int_{-\pi}^{\pi} \frac{1}{\alpha^{2 n-1}} \int_{\omega_{1}}^{\omega_{2}} \cos ^{2 n-2} \omega \mathrm{d} \omega \mathrm{d} \theta,
$$

where $\omega_{1}=-\arctan (Z / \alpha)$ and $\omega_{2}=-\arctan ([Z-L] / \alpha)$. The inner integral can be evaluated as in (3.3). Thus, $R_{2 n}$ has two forms of integral which are needed to be evaluated, namely

$$
S_{m}=\int_{-\pi}^{\pi} \frac{1}{\alpha^{m}} \arctan \left(\frac{\beta_{i}}{\alpha}\right) \mathrm{d} \theta, \quad T_{m}=\int_{-\pi}^{\pi} \frac{1}{\alpha^{k}} \frac{1}{\left[\alpha^{2}+\beta_{i}^{2}\right]^{m}} \mathrm{~d} \theta,
$$

where $\beta_{1}=Z$ and $\beta_{2}=Z-L$. We comment that the integrals in (3.9) can be evaluated numerically and their analytical expressions are given in [17].

The interaction energy for a sphere around the edge of a nanotube can be written as

$$
E_{S T}^{*}=8 \pi^{2} a b^{2} \mu_{s} \mu_{t}\left\{-A\left[V_{2}+2 b^{2} V_{3}\right]+\frac{B}{5}\left[5 V_{5}+80 b^{2} V_{6}+336 b^{4} V_{7}+512 b^{6} V_{8}+256 b^{8} V_{9}\right]\right\} \text {. }
$$

Following [18], $V_{n}$ is defined by

$$
V_{n}=\int_{-\pi}^{\pi} \frac{1}{\lambda^{2 n-1}} \int_{\omega_{1}}^{\sigma_{2}} \cos ^{2 n-2} \varpi \mathrm{d} \varpi \mathrm{d} \theta,
$$

where $\lambda^{2}=(a+x)^{2}-4 a x \cos ^{2}(\theta / 2)-b^{2}, \varpi_{1}=\arctan (Z / \lambda)$ and $\varpi_{2}=-\arctan ([Z-L] / \lambda)$. We comment that Baowan et al. [18] develop this model for a semi-infinite tube, whereas in our case this model is for a finite tube. 


\section{Numerical Results}

In this section, we present numerical results for the interaction between a chloroform molecule and a peptide nanotube. Firstly, we show the results for an on-axis chloroform molecule interacting with one peptide unit of various sizes. We plot the graph of the interaction energies versus the axial position of the chloroform molecule, $Z$. Noting that $Z=0$ denotes the position where the chloroform molecule is at the open end of the nanotube. As shown in Figure 3, there is a high energy barrier at the open end of the peptide unit of inner radius $4.25 \AA$ that prevents the chloroform molecule from being accepted into the nanotube. Figure 4 shows the energy profiles for

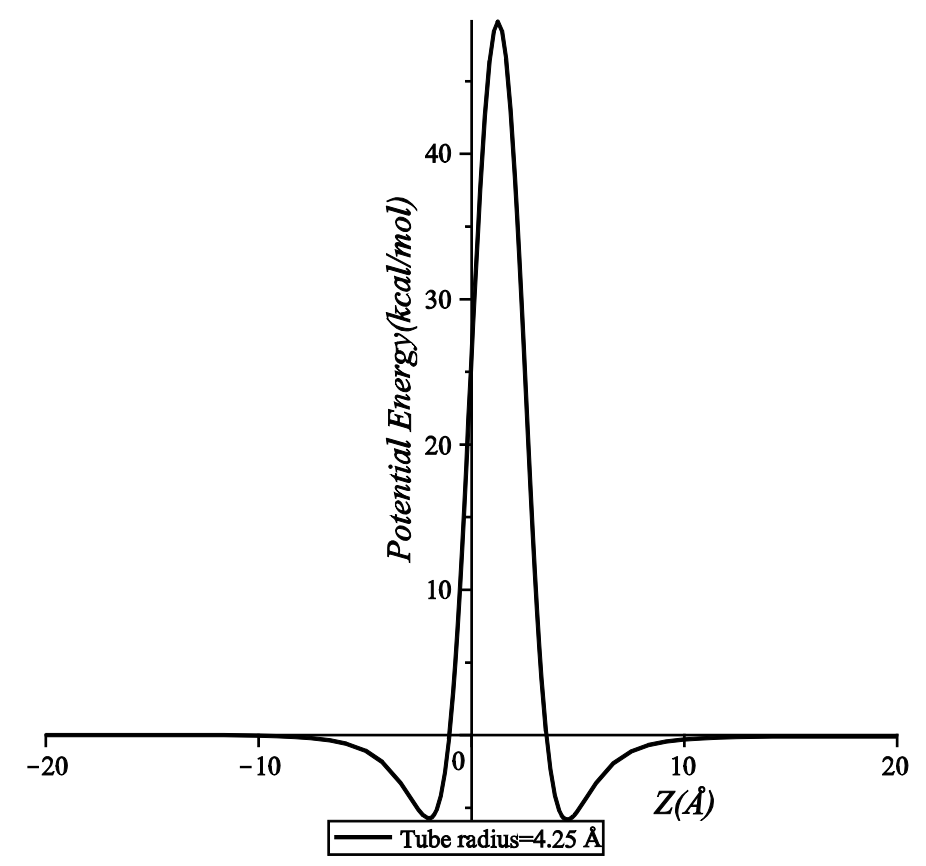

Figure 3. Potential energy for an on-axis chloroform molecule interacting with a peptide unit which has the inner radius of $4.25 \AA$.

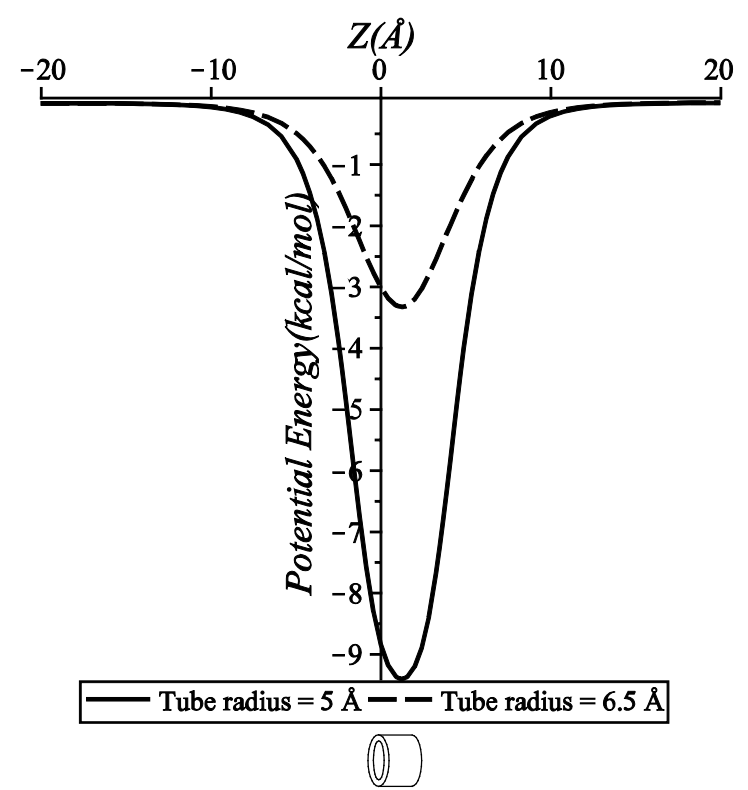

Figure 4. Potential energy for an on-axis chloroform molecule interacting with two sizes of a peptide unit (5 $\AA$ and $6.5 \AA$ inner tube radii). 
peptide nanotubes of inner radii $5 \AA$ and $6.5 \AA$. We can see that the minimum energy occurs inside the peptide unit. This result suggests that the chloroform molecule can be encapsulated into both peptide units. However, the chloroform molecule is more stable inside the $5 \AA$ peptide nanotube as the energy is much lower than that of the $6.5 \AA$ peptide nanotube. Thus, we can conclude that the encapsulation process is strongly dependent on the size of the inner radius of the peptide nanotube.

Figure 5 illustrates the strong effect of the inner tube (backbone atoms) upon the total interaction energy by plotting the interactions for: 1) the chloroform molecule and the inner tube of the peptide unit and 2) the chloroform molecule and both the inner and outer tubes of the peptide unit. We use the inner tube of radius $5 \AA$ in Figure 5. We find that there is only a slight difference between the energies for both interactions. This finding shows that the short range interaction between the chloroform molecule and the backbone atoms on the inner tube dominates the total interaction energy. This may be due to that the side chain atoms on the outer tube are further away from the chloroform molecule.

Next, we plot in Figure 6 the interaction energy between a chloroform molecule and a peptide nanotube with more than one peptide units. Again, this is the case when the inner radius of the peptide nanotube is $5 \AA$. In

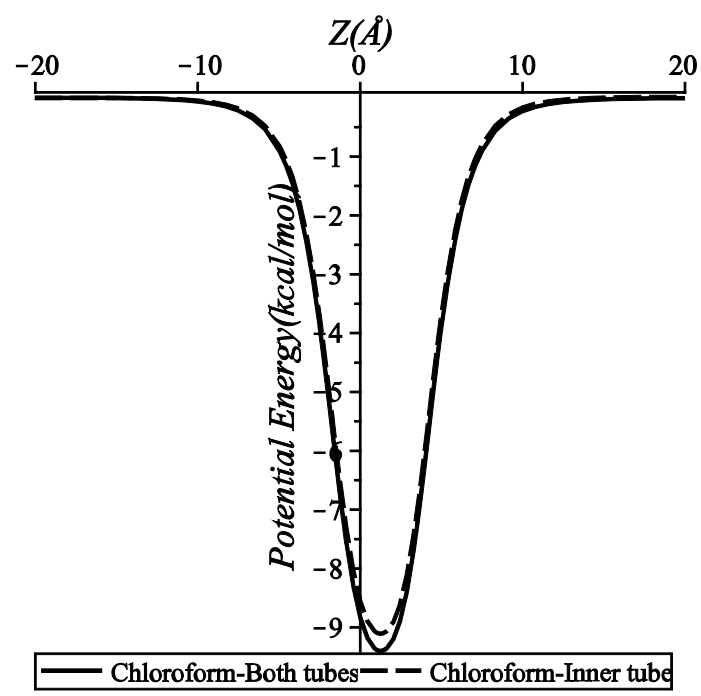

Figure 5. Interaction of a chloroform molecule-an inner tube of a peptide unit and a chloroform molecule-both inner and outer tubes of a peptide unit.

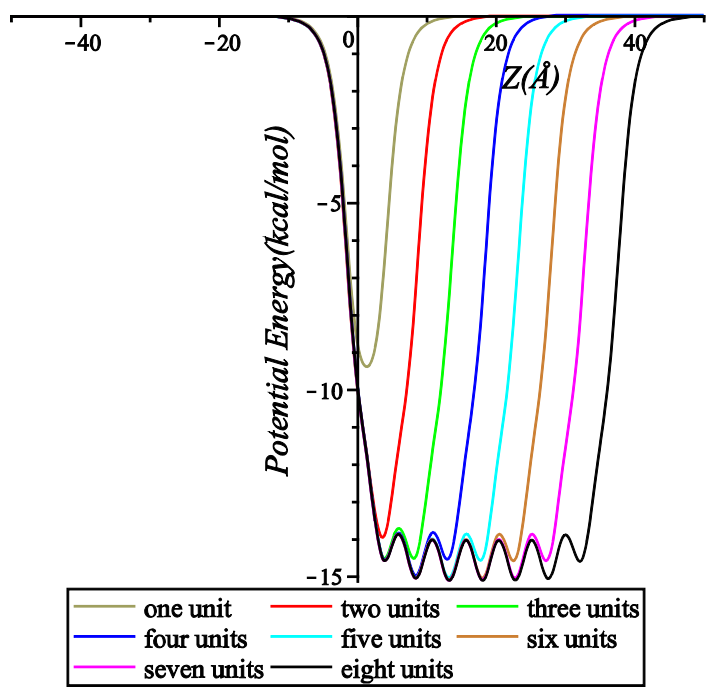

Figure 6. Interaction energy between a chloroform molecule and peptide nanotubes with various numbers of peptide units. 
Figure 6 we observe an undulating behaviour for the interactions involving at least three peptide units. For the case of four or more peptide units, the local minima reach the same value implying that additional units do not significantly affect the magnitude of the minimum energy. We conclude that using four peptide units is adequate to attain the minimum energy for this case.

Next, we determine the preferred location of the chloroform molecule inside the peptide nanotube. This position can be either within the peptide unit or in the space between each unit. As shown in Figure 7, we find that the minimum energy occurs when the chloroform molecule is located in the space between each peptide unit. This result is in good agreement with [11] which concludes that every chloroform molecule occupies the centre cavity between two neighboring units. Even though we use a simpler type of a peptide nanotube compared with that of [11], the backbone atomic structures are similar and therefore our results are comparable. In addition, given the size of the molecule, this result is consistent with the preferred location of a water molecule in a peptide nanotube [14] [15] and an ion-water cluster in a peptide nanotube [24].

In Table 2, we present the interaction energy between a peptide nanotube of inner radius $5 \AA$ and the chloroform molecule which is assumed to be located near the edge of the nanotube in the positive $z$-direction. We note

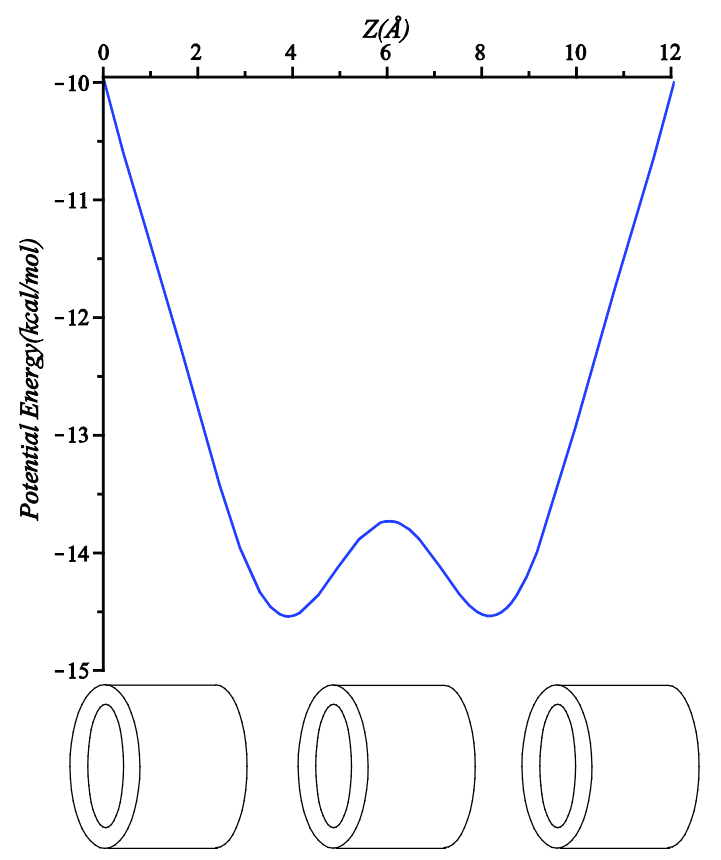

Figure 7. Interaction energy between a chloroform molecule and a nanotube with three peptide units.

Table 2. Numerical values for the minimum interaction energies for the choloform molecule locating near the edge of a peptide unit. Parameters $\varphi$ and $r$ are as shown in Figure 2.

\begin{tabular}{ccc}
\hline Angle $\varphi$ & $r(\AA)$ & $E_{\min }(\mathrm{kcal} / \mathrm{mol})$ \\
\hline$\pi / 6$ & 4.91 & -75.2343 \\
$\pi / 4$ & 4.28 & -98.6089 \\
$\pi / 3$ & 3.68 & -127.3989 \\
$5 \pi / 12$ & 3.31 & -130.5658 \\
$\pi / 2$ & 3.14 & -112.04876 \\
$7 \pi / 12$ & 3.069 & -92.6664 \\
$2 \pi / 3$ & 3.032 & -78.8921 \\
$3 \pi / 4$ & 3.014 & -70.5589 \\
\hline
\end{tabular}


that when $\varphi>\pi / 2$ the chloroform molecule is located on the left hand side closed to the open end of the peptide nanotube. From Table 2 the chloroform molecule will not be encapsulated into the tube if its location is at the edge on the positive $z$-direction of the tube. This is because of the lower energy level at that position, which is needed to be overcome before the molecule can move inside the nanotube. However, the entrance would occur if an initial energy is given for the chloroform molecule to overcome such an energy barrier. The chloroform molecule has a greater probability of encapsulation around the tube edge if it is initially located closer to the tube edge at $\varphi>5 \pi / 2$. We note that for the chloroform molecule that can overcome the energy barrier and located in the negative $z$-direction, the analysis for the encapsulation as shown the previous section applies.

\section{Summary}

This paper investigates two scenarios of a chloroform molecule interacting with a peptide nanotube by employing the Lennard-Jones potential and a continuum approach. We find that an on-axis chloroform molecule is accepted into a peptide nanotube of inner radii $5 \AA$ or $6.5 \AA$, and is rejected from a tube of inner radius $4.25 \AA$. As a result, we can say that the encapsulation of a chloroform molecule into a peptide nanotube depends strongly on the size of the molecule and the internal diameter of the peptide nanotube. We also find that inside a nanotube the chloroform molecule prefers to be in the space between each peptide unit. Further, our results suggest that the chloroform molecule located near the edge on the positive z-direction along the nanotube is unlikely to be encapsulated into the peptide nanotube. This is due to the high energy barrier that the molecule would need to overcome to move to the negative $z$-direction to be closer to the open end of the nanotube.

Our results presented here provide an insight into the encapsulation of a chloroform molecule into a peptide nanotube and its behavior inside the nanotube. Our findings support Cheng et al. [11] on the potential use of peptide nanotubes as artificial channels for transporting organic molecules across cell membranes, indicating the ability to use peptide nanotubes in applications of targeted drug and gene delivery [28].

\section{Acknowledgements}

F. R. gratefully acknowledges the Ministry of Higher Education Malaysia and the Universiti Pendidikan Sultan Idris Malaysia for the provision of a $\mathrm{PhD}$ scholarship.

\section{References}

[1] Cheng, J., Zhu, J., Liu, B., Liao, Z. and Lai, Z. (2009) Structure of a Self-Assembled Single Nanotube of Cyclo[(-D-Ala-L-Ala) $\left.4^{-}\right]$. Molecular Simulation, 35, 625-630. http://dx.doi.org/10.1080/08927020902787788

[2] Hartgerink, J.D., Granja, J.R., Miligan, R.A. and Ghadiri, M.R. (1996) Self-Assembling Peptide Nanotubes. Journal of the American Chemical Society, 118, 43-50. http://dx.doi.org/10.1021/ja953070s

[3] Garcia-Fandino, R., Castedo, L., Granja, J.R. and Vasquez, S.A. (2010) Interaction and Dimerization Energies in Methyl-Blocked $\alpha, \gamma$-Peptide Nanotube Segments. The Journal of Physical Chemistry B, 114, 4973-4983. http://dx.doi.org/10.1021/jp910919k

[4] Reches, M. and Gazit, E. (2006) Molecular Self-Assembly of Peptide Nanostructures: Mechanism of Association and Potential Uses. Current Nanoscience, 2, 105-111. http://dx.doi.org/10.2174/157341306776875802

[5] Zhu, J., Cheng, J. and Liao, Z. (2008) Investigation of Structures and Properties of Cyclic Peptide Nanotubes by Experiments and Molecular Dynamics. Journal of Computer-Aided Molecular Design, 22, 773-781. http://dx.doi.org/10.1007/s10822-008-9212-9

[6] Ghadiri, M.R., Granja, J.R. and Buehler, L.K. (1994) Artificial Transmembrane Ion Channels from Self-Assembling Peptide Nanotubes. Nature, 369, 301-304. http://dx.doi.org/10.1038/369301a0

[7] Liu, J., Fan, J., Min, T. and Zhou, W. (2010) Molecular Dynamics Simulation for the Structure of the Water Chain in a Transmembrane Peptide Nanotube. The Journal of Physical Chemistry A, 114, 2376-2383. http://dx.doi.org/10.1021/jp910624z

[8] Jishi, R.A., Braier, N.C., White, C.T. and Mintmire, J.W. (1998) Peptide Nanotubes: An Inert Environment. Physical Review B, 58, R16009. http://dx.doi.org/10.1103/PhysRevB.58.R16009

[9] Asthagiri, D. and Bashford, D. (2002) Continuum and Atomistic Modeling of Ion Partitioning into a Peptide Nanotube. Biophysical Journal, 82, 1176-1189. http://dx.doi.org/10.1016/S0006-3495(02)75475-1

[10] Hwang, H., Schatz, G.C. and Ratner, M.A. (2006) Steered Molecular Dynamics Studies of the Potential of Mean Force of a $\mathrm{Na}^{+}$and $\mathrm{K}^{+}$Ion in a Cyclic Peptide Nanotube. The Journal of Physical Chemistry B, 110, 26448-26460. 
http://dx.doi.org/10.1021/jp0657888

[11] Cheng, J., Zhu, J. and Liu, B. (2007) Molecular Modeling Investigation of Adsorption of Self-Assembled Peptide Nanotube of Cyclo-[(1 R, 3S)- $\gamma$-Acc-D-Phe $]_{3}$ in $\mathrm{CHCl}_{3}$. Chemical Physics, 333, 105-111. http://dx.doi.org/10.1016/j.chemphys.2007.01.014

[12] Dehez, F., Tarek, M. and Chipot, C. (2007) Energetics of Ion Transport in a Peptide Nanotube. Journal of Physical Chemistry B, 111, 10633-10635. http://dx.doi.org/10.1021/jp075308s

[13] Engels, M., Bashford, D. and Ghadiri, M.R. (1995) Structure and Dynamics of Self-Assembling Peptide Nanotubes and the Channel-Mediated Water Organization and Self-Diffusion. A Molecular Dynamics Study. Journal of the American Chemical Society, 117, 9151-9158. http://dx.doi.org/10.1021/ja00141a005

[14] Tarek, M., Maigret, B. and Chipot, C. (2003) Molecular Dynamics Investigation of an Oriented Cyclic Peptide Nanotube in DMPC Bilayers. Biophysical Journal, 85, 2287-2298. http://dx.doi.org/10.1016/S0006-3495(03)74653-0

[15] Raghavender, U.S., Aravinda, K.S., Shamala, N. and Balaram, P. (2009) Hydrophobic Peptide Channels and Encapsulated Water Wires. Journal of the American Chemical Society, 132, 1075-1086. http://dx.doi.org/10.1021/ja9083978

[16] Girifalco, L.A. (1991) Interaction Potential for Carbon (C60) Molecules. Journal of Physical Chemistry, 95, 5370-5371. http://dx.doi.org/10.1021/j100167a002

[17] Baowan, D. and Hill, J.M. (2007) Force Distribution for Double-Walled Carbon Nanotubes and Gigahertz Oscillators. Zeitschrift für angewandte Mathematik und Physik, 58, 857-875. http://dx.doi.org/10.1007/s00033-006-6098-z

[18] Baowan, D., Thamwattana, N. and Hill, J.M. (2007) Encapsulation of $C_{60}$ Fullerenes into Single-Walled Carbon Nanotubes: Fundamental Mechanical Principles and Conventional Applied Mathematical Modeling. Physical Review B, 76, Article ID: 155411. http://dx.doi.org/10.1103/PhysRevB.76.155411

[19] Cox, B.J., Thamwattana, N. and Hill, J.M. (2008) Orientation of Spheroidal Fullerenes inside Carbon Nanotubes with Potential Applications as Memory Devices in Nano-Computing. Journal of Physics A: Mathematical and Theoretical, 41, Article ID: 235209. http://dx.doi.org/10.1088/1751-8113/41/23/235209

[20] Cox, B.J., Thamwattana, N. and Hill, J.M. (2007) Mechanics of Atoms and Fullerenes in Single-Walled Carbon Nanotubes. I. Acceptance and Suction Energies. Proceedings of the Royal Society A, 463, 461-476.

[21] Baowan, D., Cox, B.J. and Hill, J.M. (2012) Instability of $\mathrm{C}_{60}$ Fullerene Interacting with Lipid Bilayer. Journal of Molecular Modeling, 18, 549-557. http://dx.doi.org/10.1007/s00894-011-1086-4

[22] Baowan, D. and Thamwattana, N. (2014) Modelling Encapsulation of Gold and Silver Nanoparticles inside Lipid Nanotubes. Physica A, 396, 149-154. http://dx.doi.org/10.1016/j.physa.2013.11.003

[23] Baowan, D., Peuschel, H., Kraegeloh, A. and Helms, V. (2013) Energetics of Liposomes Encapsulating in Silica Nanoparticles. Journal of Molecular Modeling, 19, 2459-2472. http://dx.doi.org/10.1007/s00894-013-1784-1

[24] Rahmat, F., Thamwattana, N. and Cox, B.J. (2011) Modelling Peptide Nanotubes for Artificial Ion Channels. Nanotechnology, 22, Article ID: 445707. http://dx.doi.org/10.1088/0957-4484/22/44/445707

[25] Granja, J.R. and Ghadiri, M.R. (1994) Channel-Mediated Transport of Glucose across Lipid Bilayers. Journal of the American Chemical Society, 116, 10785-10786. http://dx.doi.org/10.1021/ja00102a054

[26] Khazanovich, N., Granja, J.R., McRee, D.E., Milligan, R.A. and Ghadiri, M.R. (1994) Nanoscale Tubular Ensembles with Specified Internal Diameters. Design of a Self-Assembled Nanotube with a 13-.ANG. Pore. Journal of the American Chemical Society, 116, 6011-6012. http://dx.doi.org/10.1021/ja00092a079

[27] Rappe, A.K., Casewit, C.J., Colwell, K.S., Goddard III, W.A. and Skiff, W.M. (1992) UFF, a Full Periodic Table Force Field for Molecular Mechanics and Dynamics Simulations. Journal of the American Chemical Society, 114, 1002410035. http://dx.doi.org/10.1021/ja00051a040

[28] Martin, C.R. and Kohli, P. (2003) The Emerging Field of Nanotube Biotechnology. Nature Reviews Drug Discovery, 2, 29-37. http://dx.doi.org/10.1038/nrd988 
Scientific Research Publishing (SCIRP) is one of the largest Open Access journal publishers. It is currently publishing more than 200 open access, online, peer-reviewed journals covering a wide range of academic disciplines. SCIRP serves the worldwide academic communities and contributes to the progress and application of science with its publication.

Other selected journals from SCIRP are listed as below. Submit your manuscript to us via either submit@scirp.org or Online Submission Portal.
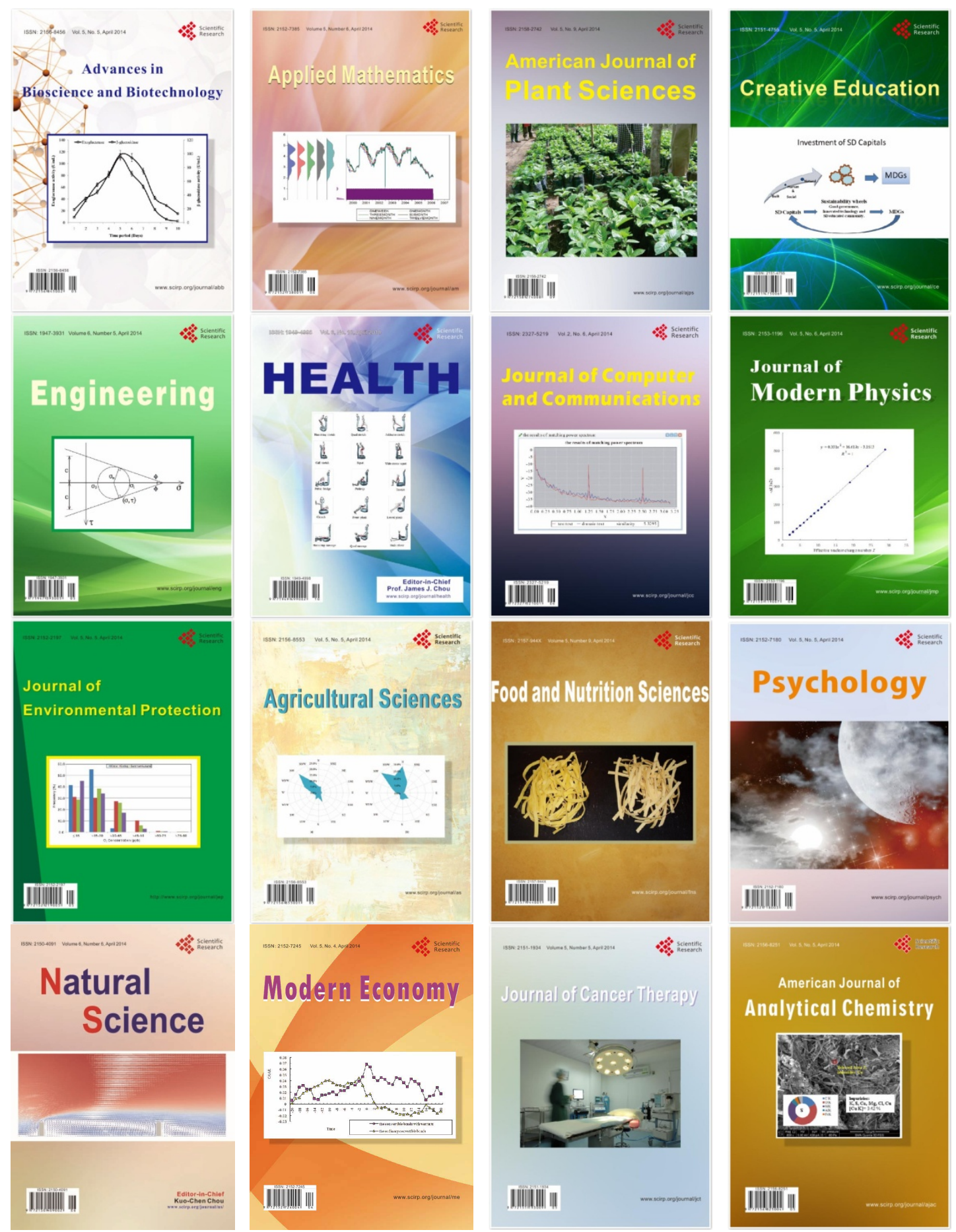